\title{
Metodologias de ensino e a promoção da inclusão de estudantes com Transtorno do Déficit de Atenção e Hiperatividade (TDAH): Uma análise em dissertações e teses da
}

\section{CAPES}

\author{
Teaching methodologies and the inclusion promotion of students with Attention Deficit \\ Hyperactivity Disorder (ADHD): An analysis in CAPES dissertations and theses \\ Metodología de enseñanza y la promoción de la inclusión de estudiantes con Trastorno de Déficit de \\ Atención e Hiperactividad (TDAH): Un análisis en disertaciones y tesis de CAPES
}

Recebido: 03/07/2021 | Revisado: 08/07/2021 | Aceito: 13/07/2021 | Publicado: 24/07/2021

\section{Resumo}

Mara Aparecida de Miranda Batista Dias ORCID: https://orcid.org/0000-0003-0976-5862 Universidade Federal do Pampa, Brasil maradias.aluno@unipampa.edu.br

Rosana Backes da Rosa ORCID: https://orcid.org/0000-0002-8165-7754 Universidade Federal do Pampa, Brasil rosanaapoteose@gmail.com

Luciana Vargas Pedroso ORCID: https://orcid.org/0000-0003-2965-3083 Universidade Federal do Pampa, Brasil E-mail: lucianapedroso@unipampa.edu.br

Edward Frederico Castro Pessano ORCID: https://orcid.org/0000-0002-6322-6416 Universidade Federal do Pampa, Brasil

E-mail: edwardpessano@unipampa.edu.br

Ailton Jesus Dinardi

ORCID: https://orcid.org/0000-0002-5625-1787 Universidade Federal do Pampa, Brasil E-mail: ailtondinardi@unipampa.edu.br

Objetivo: O presente estudo tem como objetivo analisar trabalhos que versam sobre estudantes com Transtorno de Déficit de Atenção e a proposição de metodologias de ensino que favoreçam a sua aprendizagem e inclusão. Metodologia: Trata-se de uma pesquisa bibliográfica, qualitativa e exploratória, na qual foram realizadas buscas no banco de dados do Catálogo de Teses e Dissertações da Capes utilizando para a busca o descritor "Transtorno de Déficit de Atenção e Hiperatividade". O recorte temporal utilizado nas buscas foi o período de 2015 a 2020. Para análise dos dados, teve por base a Análise de Conteúdo, de Bardin. Nesta investigação foram encontrados 160 trabalhos, sendo 121 dissertações e 39 teses, a partir da leitura dos resumos foram descartados os duplicados e os que não se referiam a área da educação, restando 25 , sendo 7 teses e 18 dissertações, estes foram lidos na íntegra excluindo os que não estavam relacionados ao docente e a prática inclusiva.Para fim deste estudo constatou-se as seguintes práticas encontradas: a) A Contribuição da Khan Academy na aprendizagem de conteúdos matemáticos, em que se percebeu que aliar jogos com exercícios corrobora para a aprendizagem; b) Aplicativo móvel para auxiliar alunos do ensino fundamental que possuem o TDAH no aprendizado da tabuada, o qual se certificou que o lúdico contribui para aproximação do conteúdo matemático básico; c) A construção da narrativa em textos produzidos por estudantes com e sem TDAH, em que se concluiu que a criatividade é fundamental para estimular a escrita. Conclusão: As práticas inclusivas encontradas neste estudo são extremamente significativas, pois indicam alternativas possíveis para a promoção da inclusão escolar, sendo necessário divulgá-las como forma de inspiração a outros docentes. Ressalta-se, ainda, que novos estudos são imprescindíveis para que a Educação Inclusiva Brasileira possa avançar.

Palavras-chave: Professores; Educação; Inclusão.

\section{Abstract}

Objective: This study aims to analyze works which deal with students with Attention Deficit Hyperactivity Disorder and the proposition of teaching methodologies which favor their learning and inclusion. Methodology: It is a bibliographical, qualitative and exploratory research,in which searches were carried out on Capes Theses and Dissertations Catalog database using the descriptor "Attention Deficit Hyperactivity Disorder" for the search. The time frame used on the searches was the period from 2015 to 2020. On this investigation, there were found 160 works, being 121 dissertations and 39 theses. For data analysis, it has had base on Bardin's Content Analysis. On this investigation, 
there has been found 160 works, from which 121 were dissertations and 39 were theses, from the abstracts readings there have been discarded duplicates and those which did not refer to the area of education, remaining 25 , from which 7 were theses and 18 were dissertations, which were read in full excluding those which were not related to the teacher and inclusive practice. For the purpose of this study there were found the following practices: a) Contribution of Khan Academy on learning mathematical content, from which we verified combining games with exercises contributes to learning; b)Mobile application to help elementary school students who have ADHD on learning multiplication table; which we certified playful activities contribute to the basic mathematical content approximation; c) The narrative construction in texts produced by students with and without ADHD in which we realized creativity is essential to stimulating writing. Conclusion: The inclusive practices found in this study are extremely significant, as they indicate possible alternatives to school inclusion promotion, being necessary to disseminate them as a way of inspiration to other teachers. We emphasize further studies are essential to Brazilian Inclusive Education to advance.

Keywords: Teacher; Education; Inclusion.

\section{Resumen}

Objetivo: El presente estudio tiene como objetivo analizar trabajos que versan sobre estudiantes con Trastorno de Déficit de Atención e Hiperactividad y la proposición de metodologías de enseñanza que favorezcan su aprendizaje e inclusión. Metodología: Se trata de una pesquisa bibliográfica, cualitativa y exploratoria, en la cual fueron realizadas búsquedas en bases de datos del Catálogo de Tesis y Disertaciones de Capes utilizando para la búsqueda el descriptor "Transtorno de Déficit de Atenção e Hiperatividade”. El recorte temporal utilizado en las búsquedas fue el período de 2015 a 2020. En esta investigación fueron encontrados 160 trabajos, siendo 121 disertaciones y 39 tesis. a partir de la lectura de los resúmenes fueron descartados los duplicados y los que no se referían al área de la educación, restando 25 , siendo 7 tesis y 18 disertaciones, estos fueron leídos integralmente siendo excluidos los que no estaban relacionados a la docencia y a la práctica inclusiva. Para fin de este estudio se constató las siguientes prácticas encontradas: a) La Contribución de Khan Academy en el aprendizaje de contenidos matemáticos, donde constatamos que aliar juegos con ejercicios corrobora para el aprendizaje; b) Aplicativo móvil para auxiliar alumnos de enseñanza fundamental que poseen TDAH en el aprendizaje de la tabla; el cual certificamos que lo lúdico contribuye para la aproximación del contenido matemático básico; c) La construcción de la narrativa en textos producidos por estudiantes con y sin TDAH donde percibimos que la creatividad es fundamental para estimular la escrita. Conclusión: Las prácticas inclusivas encontradas en este estudio son extremamente significativas, pues indican alternativas posibles para la promoción de la inclusión escolar, siendo necesario divulgarlas como forma de inspiración para otros docentes. Resaltamos que nuevos estudios son imprescindibles para que la Educación Inclusiva Brasileña pueda avanzar.

Palabras clave: Profesores; Educación; Inclusión.

\section{Introdução}

A educação inclusiva no Brasil tem início a partir da promulgação da Constituição de 1988, porém o instrumento legal central para a educação nacional está fundamentado na Lei de Diretrizes e Bases da Educação Nacional-LDBEN n. 9.394 (Brasil, 1996), de 20 de dezembro de 1996, a qual promoveu um avanço qualitativo na concepção e diretrizes da educação reforçando um caráter humanista e social, com destaque à formação do cidadão em relação a seu pleno exercício no espaço socioeducativo. Outro importante instrumento legal se refere ao Estatuto da Criança e do Adolescente - Lei ${ }^{\circ}$. 8.069/90, que reforça e tece artigos que asseguram proteção às crianças e adolescentes.

No âmbito internacional, diversas conferências começam a repercutir diretamente na educação inclusiva: no ano de 1990 ocorreu a Conferência Mundial sobre Educação para Todos, em Jomtien, na Tailândia, em 1994 em Salamanca na Espanha, acontece a Conferência Mundial sobre Necessidades Educativas Especiais, em 2008 ocorreu a $48^{\mathrm{a}}$ Conferência Internacional sobre Educação da UNESCO em Genebra, tendo a Educação Inclusiva como principal tema.

No Brasil, estas recomendações internacionais embasaram a criação de políticas públicas visando a inclusão escolar, embora somente no ano de 2015 foi instituída a Lei Brasileira de Inclusão - Lei 13.146/2015 - criada em 6 de julho de 2015 e aprovada em 2 de janeiro de 2016, também conhecida como Estatuto da pessoa com deficiência, é um dos mais novos avanços na luta em favor da inclusão social e cidadã.

A Lei Brasileira de Inclusão da Pessoa com Deficiência dá orientações no Art. $2^{\circ}$.

Art. 2o Considera-se pessoa com deficiência aquela que tem impedimento de longo prazo de natureza física, mental, intelectual ou sensorial, o qual, em interação com uma ou mais barreiras, pode obstruir sua participação plena e efetiva 
na sociedade em igualdade de condições com as demais pessoas.

1o A avaliação da deficiência, quando necessária, será biopsicossocial, realizada por equipe multiprofissional e interdisciplinar e considerará: I - os impedimentos nas funções e nas estruturas do corpo; II - os fatores socioambientais, psicológicos e pessoais; III - a limitação no desempenho de atividades; e IV - a restrição de participação.

2o. O Poder Executivo criará instrumentos para avaliação da deficiência. (Brasil, 2015)

A mudança de perspectiva sobre a palavra "deficiência" é uma das vantagens da Lei Brasileira de Inclusão. A deficiência era vista, anteriormente, como uma condição da pessoa. A partir da lei, passa a ser entendida como uma situação dos espaços (físicos ou sociais) que não estão prontos para recebê-las. (LBI, 2015).

Outro ponto a ressaltar é a contemplação ou não na Legislação Brasileira das pessoas com transtorno do neurodesenvolvimento, por exemplo o Transtorno de Déficit de Atenção (TDAH), objeto deste estudo, visto que especificamente nominalmente na Lei está descrito somente como categoria, o Autismo (categoria VI da descrição de deficiência), conforme descrição citada a seguir:

Considera-se deficiência toda restrição física, intelectual ou sensorial, de natureza permanente ou transitória, que limita a capacidade de exercer uma ou mais atividades essenciais da vida diária e/ou atividades remuneradas, causada ou agravada pelo ambiente econômico e social, dificultando sua inclusão social, enquadrada em uma das seguintes categorias: I - deficiência física, II - deficiência auditiva, III - deficiência visual, IV - deficiência intelectual, V surdocegueira, VI - autismo, VII - condutas típicas, VIII - deficiência múltipla.” (Brasil, 2015)

Sendo assim, entende-se que as crianças que possuem TDAH são submetidas à avaliação pelo Poder Executivo, para serem incluídas na escola e gozar de seus direitos descritos na Lei Brasileira de Inclusão. (Brasil, 2015).

Em relação à legislação voltada para educação brasileira, tem-se a proposta da Base Nacional Comum Curricular (BNCC, Brasil 2018), a qual está voltada para uma educação democrática, justa e inclusiva. Sendo assim, deve contribuir para um avanço na educação inclusiva. $\mathrm{O}$ documento amplia as possibilidades para que as escolas procurem soluções e inovações para ensinar a todos, potencializando os indivíduos e para que ocorra uma educação inclusiva é preciso pensar no aluno como um todo. Nesse ponto, a garantia da aprendizagem das competências cognitivas e socioemocionais podem ser um ganho importante para o estudante com deficiência. A Competência 4 (quatro) da BNCC, descrita a seguir, é um exemplo claro da proposta inclusiva.

Prevê utilizar diferentes linguagens - verbal (oral ou visual-motora, como libras e escrita) corporal, visual, sonora e digital - bem como conhecimentos das linguagens artística, matemática e científica para expressar e partilhar informações, experiências, ideias e sentimentos em diferentes contextos e produzir sentidos que levem ao entendimento mútuo. (Brasil, 2018)

Nessa perspectiva inclusiva, os professores são a peça chave para que este atendimento seja desempenhado com eficácia, visto que, o planejamento para atender os estudantes inclusos proporcionam o desenvolvimento do processo de inclusão. A aplicação de estratégias pedagógicas diferenciadas pode ser utilizada como um recurso que possibilita a aprendizagem de todos os estudantes. Conforme Braccialli e Paiva (2010) e Manzini e Santos (2002), as estratégias diferenciadas podem estar relacionadas a recursos adaptados de acordo com a deficiência específica do estudante de uma forma simples para a rotina escolar ou ainda podendo ser complexa como o uso de recursos digitais, por exemplo. Para tanto, as estratégias estão relacionadas com a forma de oferecer ao estudante oportunidades para estabelecer situações de ensino-aprendizagem, tais como: planejamento da aula, apresentação de ideias, debates, dramatização, pesquisas, projetos, estudo dirigido, estudo do meio, seminários, trabalhos em duplas ou em grupo.

Nesse contexto, o foco deste estudo está direcionado para o Transtorno de déficit de atenção e hiperatividade (TDAH) o qual está definido como um transtorno neurobiológico, de causas genéticas, que aparece na infância e frequentemente 
acompanha o indivíduo por toda a sua vida. Os sintomas presentes são desatenção, inquietude e impulsividade; às vezes, chamado de Distúrbio do déficit de atenção (DDA). Em inglês, também é chamado de ADD, ADHD ou de AD/HD, conforme a Associação Brasileira de Déficit de Atenção (Associação Brasileira do Déficit de Atenção [ABDA], 2019). O transtorno é reconhecido oficialmente por vários países e pela Organização Mundial da Saúde (OMS).

Rodhe et al. (2003) afirmam que esse transtorno é um dos mais comuns na infância, o que, segundo dados, ocorre com maior frequência em crianças entre 3 a $5 \%$ dessa população infantil. A criança que apresenta perfil de comportamento com ausência de atenção e concentração, hiperatividade e/ou impulsividade, em grau maior que aquele observado entre a mesma faixa etária e período de desenvolvimento, enquadra-se conforme DSM IV e V1 e OMS2, com perfil de hiperatividade ou transtorno hipercinético.

Sendo assim, este estudo objetiva o propósito de investigar quais são as estratégias utilizadas pelos professores para desenvolverem a aprendizagem dos estudantes que apresentam o transtorno.

Para um diagnóstico do TDAH, é preciso levar em consideração a idade anterior aos 7 anos e o comportamento em dois ambientes; por exemplo, o comportamento tanto na escola como no convívio familiar por um período de seis meses. Conforme Rohde et al. (2008), é necessário a observação do padrão de comportamento hiperativo e sintomas de desatenção e hiperatividade com frequência como:

[...] frequentemente dá respostas precipitadas antes das perguntas terem sido concluídas; ter dificuldade em esperar a sua vez; interromper ou se meter em assuntos de outros; agitar as mãos ou os pés ou se remexer na cadeira; abandonar sua cadeira em sala de aula ou outras situações nas quais se espera que permaneça sentado; correr ou escalar em demasia, em situações nas quais isto é inapropriado; pela dificuldade em brincar ou envolver-se silenciosamente em atividades de lazer; estar frequentemente "a mil" ou muitas vezes agir como se estivesse "a todo o vapor"; e falar em demasia. (Rohde et al., 2008, p. 2).

Nesta conjuntura, Rohde et al. (2008) aponta que a criança diagnosticada com TDAH raramente escuta as instruções e finaliza as atividades solicitadas pelos pais ou professores. No entanto, ela também pode demonstrar interesse por tudo o que está acontecendo no ambiente ao seu redor, embora não sustente esse interesse, principalmente quando julgar as atividades desagradáveis. Sendo assim, esses sintomas frequentemente afetam a vida escolar e as interações sociais dos indivíduos com TDAH (AAP, 2019).

Nesse sentido, a caracterização dos sintomas de déficit de atenção, hiperatividade e impulsividade, conforme Mattos (2003), pode ser classificado em quatro modalidades descritas a seguir: perfil desatento - não enxerga detalhes, comete erros por falta de cuidado, apresenta dificuldade em manter a atenção, parece não ouvir, tem dificuldade em seguir instruções, desorganização, evita/não gosta de tarefas que exigem um esforço mental prolongado, distrai-se com facilidade, esquece atividades diárias; perfil hiperativo/impulsivo - apresenta uma inquietação constante, mexe as mãos e os pés, remexe-se na cadeira, dificuldade em permanecer sentado, corre sem destino, sobe nos móveis ou muros, dificuldade em engajar-se numa atividade silenciosa, fala excessivamente, responde perguntas antes de elas serem formuladas, interrompem assuntos que estão sendo discutidos e se intrometem nas conversas; perfil combinado - quando a pessoa apresenta concomitantemente os dois conjuntos de critérios desatento e hiperativo/impulsivo; perfil não específico - quando as características apresentadas são insuficientes para se chegar a um diagnóstico completo, apesar dos sintomas desequilibrar a rotina diária. Sendo assim, o diagnóstico precoce do distúrbio de aprendizagem é fundamental para a superação da dificuldade, verificando a área mais comprometida e encaminhando para o procedimento terapêutico mais adequado (Borges, 2005).

Para boa parte dos professores, O TDAH ainda é um assunto desconhecido, as informações necessitam de embasamento teórico, como causas, idade de manifestação, médico especialista e tratamento. Essas orientações estão distantes dos docentes que, muitas vezes, lecionam exatamente para esses alunos (Borges, 2005).

No âmbito da educação, significa que as metodologias, espaços e materiais devem ser capazes de atender a todos os 
estudantes da sala de aula, em uma classe inclusiva com estudantes com e sem deficiência, metodologias diferenciadas se tornam estímulos para um ambiente educativo.

Diante do que foi exposto, o presente trabalho busca, a partir de dissertações e teses disponíveis no Banco de dados do Catálogo de Teses e Dissertações da Capes (Coordenação de Aperfeiçoamento de Pessoal de Nível Superior), analisar trabalhos que versam sobre estudantes com TDAH e a proposição de metodologias de ensino que favoreçam a sua inclusão e aprendizagem.

\section{Metodologia}

O presente estudo se caracteriza como uma pesquisa bibliográfica, qualitativa e exploratória. Considera-se uma pesquisa exploratória aquela que visa proporcionar uma maior familiaridade com o problema de um tema, ainda pouco conhecido (Gil, 2008), bibliográfica, pois se baseia em produções acadêmicas ou técnicas, como livros, artigos, monografias, dissertações e/ou teses (Gil, 2008), e qualitativa, que permite que os pesquisadores usem a criatividade e imaginação, propondo uma nova ótica para o trabalho.

O período que caracterizou o recorte temporal deste trabalho foi do ano de 2015 a 2020, em virtude da entrada em vigor da Lei Brasileira de Inclusão (LBI), também chamada de Estatuto da Pessoa com Deficiência (Lei 13.146/2015), que afirmou a autonomia e a capacidade desses cidadãos para exercerem atos da vida civil em condições de igualdade com as demais pessoas (Brasil, 2015). Nesse sentido, o período de análise relacionado nos últimos 5 anos, a partir da Lei da inclusão, busca verificar as possíveis investigações acerca de metodologias de ensino propostas para a promoção da inclusão, em estudantes com TDAH.

Para a coleta de dados no Banco do Catálogo de Teses e Dissertações da Capes, inicialmente foram aplicados os seguintes filtros: a) Trabalhos desenvolvidos entre 2015 e 2020; b) Trabalhos caracterizados como Dissertações e Teses; c) Trabalho que apresentavam em seu corpo o descritor: "Transtorno de Déficit de Atenção e Hiperatividade"

Posteriormente, foi efetuado o download das produções encontradas sendo separados entre dissertações e teses. Nessa investigação, foram encontrados 160 trabalhos, sendo 121 dissertações e 39 teses. De posse dessas produções, primeiramente, foi feita uma seleção pelo título das obras, a fim de verificar quais dissertações e teses se enquadravam no objetivo da pesquisa, ou seja, referem-se à inclusão de estudantes com TDAH na escola.

Cada trabalho teve o seu resumo e suas conclusões lidas e, quando identificado que se tratava de um estudo voltado para a área da saúde (medicina, tratamentos, medicamentosos, psicologia, fisioterapia, etc.), foi descartado, bem como os trabalhos duplicados. No entanto, quando as investigações estavam relacionadas à Educação, essas foram selecionadas, lidas e analisadas novamente. Foram selecionados nesta fase 25 trabalhos, sendo 7 teses e 18 dissertações.

Após a seleção dos trabalhos, foram excluídos os que não estavam relacionados diretamente com o docente e a prática inclusiva. Nessa exclusão, foram inseridos estudos relacionados aos seguintes temas: práticas educativas dos responsáveis; a condição financeira das famílias dos estudantes; relacionamento das famílias com a escola, aplicação de testes de memórias em crianças com TDAH; análise do grupo social em que o estudante está inserido (família, profissionais da saúde, professores); comparativo fonológico; diferentes formas de escrita entre crianças com TEA, dislexia e com TDAH, associação do transtorno com aumento de peso corporal; formação continuada dos professores; percepção de alunos sem laudos diagnósticos; políticas educacionais, os efeitos da medicalização em relação ao processo de aprendizagem.

Com o intuito de atingir o objetivo proposto nessa etapa do estudo, foram contempladas 02 teses e 01 dissertação, as quais se referem a práticas educativas construídas por docentes como forma de promover a aprendizagem de estudantes com TDAH em sala de aula.

Para análise de dados, teve por base a Análise de Conteúdo, de Bardin (2011). 


\section{Resultados e Discussão}

Dentre as 160 teses e dissertações produzidas no período de 2015 a 2020, relacionadas ao TDAH, somente 03 dessas estavam relacionadas com o objetivo dessa investigação, o qual concerne em buscar pesquisas que propuseram metodologias de ensino na promoção da inclusão de estudantes com TDAH.

A seguir, apresenta-se o quadro com os resultados obtidos a partir da análise das dissertações e teses para esta pesquisa.

Quadro 1 - Estudos selecionados, 2021.

\begin{tabular}{|c|l|c|l|}
\hline Período & \multicolumn{1}{|c|}{ Autor (a) } & $\begin{array}{c}\text { Tipo de } \\
\text { Estudo }\end{array}$ & \multicolumn{1}{c|}{ Título } \\
\hline 2016 & Alexandre Matias Russo & Dissertação & $\begin{array}{l}\text { A contribuição da khan academy na aprendizagem de conteúdos } \\
\text { matemáticos: uma proposta para alunos com transtorno de déficit de } \\
\text { atenção e hiperatividade -TDAH }\end{array}$ \\
\hline 2016 & Wagner Marcelo Sanchez & Tese & $\begin{array}{l}\text { Aplicativo móvel para auxiliar alunos do ensino fundamental } \\
\text { portadores de TDAH no aprendizado da tabuada. }\end{array}$ \\
\hline 2018 & $\begin{array}{l}\text { Renata Amaral De Matos } \\
\text { Rocha }\end{array}$ & Tese & $\begin{array}{l}\text { A construção da narrativa em textos produzidos por estudantes com e } \\
\text { sem TDAH. }\end{array}$ \\
\hline
\end{tabular}

Fonte: Autores.

Nesse propósito, expõe-se as práticas encontradas neste estudo para promover a aprendizagem em alunos com TDAH no contexto da sala de aula inclusiva: I) A Contribuição da Khan Academy na aprendizagem de conteúdos matemáticos; II) Aplicativo móvel para auxiliar alunos do ensino fundamental portadores de TDAH no aprendizado da tabuada; III) A construção da narrativa em textos produzidos por estudantes com e sem TDAH.

\section{I) A Contribuição da Khan Academy na aprendizagem de conteúdos matemáticos.}

Esta dissertação objetivou verificar se os conteúdos matemáticos propostos pudessem ter um aperfeiçoamento por meio de uma intervenção tecnológica, mais especificamente se a Khan Academy poderia ser um instrumento auxiliar para essa intervenção e se colaboraria para o processo de aprendizagem de alunos diagnosticados com TDAH.

A Khan Academy é uma Organização Não Governamental, sem fins lucrativos, que tem como objetivo principal a missão idealizada pelo seu fundador Salman Khan: "prover uma educação de nível internacional gratuita para qualquer um, em qualquer lugar”. (Khan, 2013). Os conteúdos de diversas áreas do conhecimento são disponibilizados no endereço eletrônico https://www.khanacademy.org, com acesso totalmente livre.

Nesse estudo, a partir da metodologia utilizada, obteve-se um resultado satisfatório quando se percebeu que os alunos, quando conectados ao banco de questões da plataforma Khan Academy, ficavam concentrados à tela do computador, resolvendo os desafios disponibilizados aleatoriamente, demonstrando interesse em superá-los e sendo estimulados a atingirem uma sequência de respostas corretas e, sempre que se fez necessário, utilizaram as interatividades para compreensão dos conteúdos propostos.

Segundo Abrahão e Fantacini (2017), o desempenho escolar, bem como a aprendizagem de alunos com TDAH, vem sendo muito defasados, tendo em vista a falta de concentração e a euforia dos estudantes que não conseguem acompanhar as atividades propostas pelo professor em sala de aula. Assim, essas interações com o ambiente da plataforma, talvez sejam, dentro dessa intervenção tecnológica, as principais medidas de contribuição para o aprimoramento dos conhecimentos matemáticos propostos e para a aprendizagem desses escolares, pois assim os alunos demonstram um engajamento com resultados positivos para a aprendizagem. 
Considera-se que os protocolos das resoluções realizados pelos alunos nas atividades podem contribuir para práticas pedagógicas que visam ao aprimoramento do ensinar Matemática para alunos com TDAH, refinando algumas e formalizando outras práticas. As ações dos alunos diagnosticados com TDAH, por meio das interações com o ambiente da plataforma, permitiram resgatar, compreender e aprimorar os temas estudados e contribuíram para o aprimoramento dos conhecimentos matemáticos propostos e para a aprendizagem desses escolares.

Para Santos e Albuquerque (2019), a utilização de computadores tem sido um instrumento proposto para o estímulo do desenvolvimento, da atenção e da concentração de estudantes, promovendo uma melhora no desempenho acadêmico e atenuando as dificuldades relativas à atenção, corroborando com os dados da pesquisa anterior.

\section{II) Aplicativo móvel para auxiliar alunos do ensino fundamental portadores de TDAH no aprendizado da tabuada}

O objetivo do desenvolvimento deste aplicativo se deteve em aumentar a eficiência na aprendizagem da tabuada, a qual se faz presente nestas circunstâncias e assim contribuir para a inclusão de alunos do ensino fundamental diagnosticados com TDAH.

A constante interferência na aprendizagem da matemática relacionada ao transtorno está descrita por Sanchez (2004), onde indica que assim se apresenta:

Em caso de presença de algum déficit sensorial, as dificuldades matemáticas ultrapassam aquelas que geralmente estão associadas. Diversas habilidades podem estar prejudicadas nesse Transtorno, como as habilidades linguísticas (compreensão e nomeação de termos, operações ou conceitos matemáticos, e transposição de problemas escritos ou aritméticos, ou agrupamentos de objetos em conjuntos), de atenção (copiar números ou cifras, observar sinais de operação e matemáticas (dar sequência a etapas matemáticas, contar objetos e aprender tabuadas de multiplicação). (Sanchez, 2004, p.177)

Em razão da dificuldade destes estudantes com TDAH na matemática esse estudo, constatou-se que a aplicação do software contribuiu para um aprendizado mais eficiente, colaborando com a linha que o ensino lúdico e prazeroso tem alto poder de acrescentar eficiência no aprendizado. Atrelada a essa contribuição pedagógica, tem-se a questão da mobilidade, que, por se tratar de um jogo para dispositivos móveis, está totalmente alinhada às expectativas das novas gerações. As novas gerações possuem modelos mentais que esperam da escola e dos educadores formas de ensinar digitais com alta conectividade e mobilidade, pois somente dessa forma poder-se-á atingir altos índices de eficiência na transmissão dos conteúdos acadêmicos.

\section{III) A construção da narrativa em textos produzidos por estudantes com e sem TDAH.}

$\mathrm{Na}$ construção desta tese, o objetivo estava ligado à investigação de desempenho linguístico de estudantes com e sem TDAH na construção de seus textos narrativos escritos, os quais foram produzidos a partir do vídeo curta-metragem de animação A ilha, de Alê Camargo, e desafiados que recontassem a história retratada no vídeo por meio de um texto narrativo escrito, em que os alunos realizaram uma retextualização, implicando tanto na leitura quanto na escrita.

Os textos produzidos pelos alunos foram o locus de observação, o que possibilitou observar marcas de desempenho desses alunos na atividade de produção textual, buscando evidências para compreensão de possíveis diferenças de desempenho entre os dois grupos de alunos, no que se refere a alguns aspectos relacionados à construção do texto: estrutura narrativa, percepção de elementos do texto fonte, universo de referência, unidade temática, progressão textual, propósito comunicativo, relevância informativa, relação entre textos.

O processo de aquisição da leitura e escrita exige itens como: atenção, concentração, memorização e organização, que estão ausentes ou prejudicados na criança com TDAH. Conforme Sampaio (2011, p. 96), a criança com transtorno tem 
dificuldade em realizar planejamentos, pois há uma disfunção no lóbulo frontal que é a área responsável pela realização dos planos e dos programas das ações humanas, bem como pela regulação e controle do comportamento.

O resultado deste estudo sugere que a criatividade e a inventividade são habilidades realçadas nos sujeitos com TDAH; por isso, os indivíduos com TDAH costumam agir com maior espontaneidade, explorar o que está a sua volta e prestar atenção em elementos que outros, sem o transtorno, talvez deixem passar despercebidos.

Assim, esta pesquisa apresenta considerações relevantes sobre o estudante que possui TDAH e aponta para um aspecto que, talvez, possa "enviesar" os diagnósticos do Transtorno: o ponto de observação. O comportamento e o desempenho dos estudantes que possuem este Transtorno são contrastados com os padrões tradicionais socialmente estabelecidos como modelos inequívocos de convivência (modelo de escola, de sala de aula, de organização, de família, de trabalho e etc), como se os padrões tradicionais fossem os únicos possíveis, considerando pouco a diversidade que há nos sujeitos e nos contextos sociais, quando, na verdade, os sujeitos precisam ser reconhecidos nas suas diferenças para que possam crescer e desenvolver todo o seu potencial.

\section{Considerações Finais}

O número de dissertações e teses produzidas relacionadas ao tema da Educação Inclusiva, mais especificamente ao Transtorno de Déficit de Atenção e Hiperatividade, contemplando a promoção de práticas inclusivas desenvolvidas por professores para a promoção da aprendizagem de estudantes com deficiência, apontam que a Educação Inclusiva no Brasil caminha a passos lentos.

As práticas inclusivas encontradas neste estudo são muito significativas e de extrema importância, pois indicam alternativas possíveis para a promoção da Inclusão escolar.

Aliar jogos com exercícios matemáticos é uma estratégia fascinante para os estudantes com deficiência e sem deficiência, pois parte da realidade digital da nossa contemporaneidade para a promoção da aprendizagem. Acredita-se que essa prática desperta o gosto e interesse pela realização das atividades pelos estudantes.

O ensino através do lúdico se torna uma possibilidade prazerosa para o estudante, tornando a prática eficiente para desenvolver a aprendizagem. Assim, a aplicação do software contribuiu para aproximação do estudante com o conteúdo matemático básico e necessário para a sequência didática do componente curricular.

Para desenvolver a escrita, faz-se necessário estimular o estudante. A criatividade utilizada para motivação da construção do texto foi o ponto alto dessa prática, a qual trouxe resultados excelentes para os estudantes que foram desafiados a realizar a proposta sugerida de forma significativa.

As estratégias de ensino, apontadas neste estudo, desenvolvidas para a promoção da aprendizagem de alunos com TDAH levam à reflexão do quão é possível utilizar outros métodos pedagógicos, além dos habituais, para desafiar os estudantes incluídos a se permitirem a buscar novas formas de aprendizagem a partir da sua vivência e dos recursos digitais disponíveis em nosso meio.

Assim sendo, acredita-se que é preciso divulgar estas práticas como forma de inspiração a outros professores e contribuição para a promoção da inclusão dos estudantes com deficiência. Outro ponto a ressaltar é que novos estudos serão sempre imprescindíveis para que a Educação Inclusiva Brasileira possa avançar.

\section{Referências}

Associação Brasileira do Déficit de Atenção. (2019). O que é TDAH. http://tdah.org.br/sobre-tdah/o-que-e-tdah.

Amaral, A. B. do et al. (2013). A formação do professor para trabalhar com crianças que apresentam diagnóstico de TDAH no ensino fundamental I na rede municipal de ensino de Curitiba. Revista Eletrônica do Curso de Pedagogia das Faculdades OPET, dez. 
Borges, S. M. C. (2005). Caminhos da leitura: análise das dificuldades e possibilidades de leitura de alunos portadores do transtorno de déficit de atenção/hiperatividade (TDAH) [Tese de Doutorado, Universidade Federal do Ceará].

Braccialli, L. M. P., \& Paiva, P. C. (2010). Textura do recurso pedagógico e implicações em atividades de encaixe realizadas por indivíduos com Paralisia Cerebral. Revista Brasileira de Educação Especial, 15(2), 1.

Brasil. (1988). Constituição da República Federativa do Brasil de 1988. http://portal.mec.gov.br/cne/arquivos/.

Brasil. (2021). Decreto N 5.296 de 2 de dezembro de 2004. http://www.planalto.gov.br/ccivil_03/_Ato2004-2006/2004/Decreto/D5296.htm.

Brasil. (2015). Lei no 13.146, de 6 de julho de 2015. Institui a Lei Brasileira de Inclusão da Pessoa com Deficiência (Estatuto da Pessoa com Deficiência). Diário Oficial da União, Brasília, DF, 7 jul. 2015. Seção 1, p. 2.

Brasil. (1996). Lei $n^{\circ} 9394$, de 20 de dezembro de 1996 (Estabelece as diretrizes e bases da Educação Nacional). <http://www.planalto.gov.br/ccivil03/Leis/19394.>.

Brasil. (1990). Lei no 8.069, de 13 de julho de 1990. Dispõe sobre o Estatuto da Criança e do Adolescente e dá outras providências. Diário Oficial [da] República Federativa do Brasil, Brasília, DF, 16 jul. 1990. <http://www.planalto.gov.br/ccivil_03/LEIS/L8069.htm\#art266>.

Brasil. (2015). Presidência da República. Secretaria-Geral. Subchefia para Assuntos Jurídicos. Lei nº 13.146, de 6 de julho de 2015. Institui a Lei Brasileira de Inclusão da Pessoa com Deficiência (Estatuto da Pessoa com Deficiência).

Brasil. (2018). Ministério da Educação. Base Nacional Comum Curricular. Brasília.

GIL, A. C. (2008). Como elaborar projetos de pesquisa (4a ed.). Atlas.

Khan, Salman. (2021). Um mundo uma escola: A educação reinventada. Intrínseca, 2013. 272 p. Edição digital. <http://lelivros.red/book/download-um-mundouma-escola-salman-khan-em-epub-mobi-e-pdf/>.

Manzini, E. J., \& Santos, M. C. F. (2002). Portal de ajudas técnicas para educação: equipamento e material pedagógico para educação, capacitação e recreação da pessoa com deficiência física: recursos pedagógicos adaptados. MEC: SEESP, fascículo 1.

Mattos, P. (2003). No mundo da lua: Perguntas e respostas sobre transtorno do déficit de atenção com hiperatividade em crianças, adolescentes e adultos. Lemos Editorial.

Rohde, L. A., \& Mattos, P. et. al. (2003). Princípios e práticas em transtorno de déficit de atenção/hiperatividade. Artes Médicas.

Rhode, A. L., \& Helpern, Ricardo. (2008). Transtorno de Déficit de atenção: Atualização. <https://www.scielo.br.>

Rohde, L. A., \& Mattos, P. et al. (2004). Princípios e práticas em TDAH.

Rocha, R. A.de M. (2018). A Construção da narrativa em textos produzidos em estudantes com e sem TDAH [Tese de doutorado, Universidade Federal de Minas Gerais, Faculdade de Letras].

Russo, A. M. (2016). A contribuição da Khan Academy na aprendizagem de conteúdos Matemáticos: uma proposta para alunos com Transtorno de Déficit de Atenção e Hiperatividade- TDAH. 193f. [Dissertação de Mestrado em Educação Matemática - Programa de Estudos Pós-Graduados em Educação Matemática, Pontifícia Universidade Católica de São Paulo].

Sampaio, S., \& Freitas, I. B de (Orgs.). (2011). Transtornos e dificuldades de aprendizagem: entendendo melhor os alunos com necessidades educativas especiais. Rio de Janeiro: Wak editora.

Sanchez, J. N. G. (2004). Dificuldades de Aprendizagem e Intervenção Psicopedagógica. Artmed.

Sanchez, W. M. (2016). Aplicativo móvel para auxiliar alunos do ensino fundamental portadores de TDAH no aprendizado da tabuada. [Tese de Doutorado em Engenharia Biomédica, Universidade de Mogi das Cruzes].

Santos, W. M., \& Albuquerque, A. R.de (2019). Intervenções escolares para o TDAH: Uma revisão da literatura (2000-2018). Psicologia: Teoria e Prática, 21(3).

Silva Abrahão, N., \& Fantacini, R. A. F. (2017). Transtorno do Déficit de Atenção com Hiperatividade (TDAH): desafios e possibilidades frente a sala de aula. Research, Society and Development, 6(3), 222-236. 